\title{
Tecnologías de la Información y Comunicación en el Desarrollo de las Competencias Matemáticas en la Educación Virtual Universitaria
}

\author{
Julio Núñez Cheng \\ junuche@hotmail.com \\ Estudiante de Doctorado de la Escuela de Postgrado \\ Universidad Cesar Vallejo UCV \\ Chimbote- Perú \\ Orcid: 0000-0002-5420-0572
}

\section{RESUMEN}

El Tratamiento de la información y competencia digital forma parte de una de las competencias que se debe lograr en los estudiantes, que consiste en disponer de una serie de habilidades para buscar, obtener, procesar y comunicar información, y se incluya las nuevas tecnologías de la información y comunicación, extrayendo su máximo rendimiento a partir de su comprensión, y donde la presente investigación tiene por objetivo, analizar el uso de herramientas tecnológicas en el logro de las competencias matemáticas en la educación virtual universitaria, utilizándose la vigilancia tecnológica en la búsqueda de información actualizada, filtrándose en periodos más cortos, el uso del software Mendeley Desktop como herramienta de compartición de documentos analizados; siendo la técnica del fichaje y las fichas sincréticas como instrumentos de procesamiento de la información, de donde se infiere que las TICS han permitido un cambio sustancial en el sector educativo como un ente facilitador del conocimiento con metodologías participativas, uso de plataformas educativas integrando estrategias tecnológicas innovadoras, que han logrado impactar en la formación de calidad del estudiante, cambiando su comportamiento cultural y social, logros en la investigación rápida y oportuna al fortalecer su aprendizaje, habilidades de búsqueda, canales de comunicación, interactividad y ahorro de tiempo.

Palabras clave: competencia; comunicación; información; matemática; tecnologías. 


\title{
Information and Communication Technologies in the Development of Mathematical Competences in Virtual University Education
}

\begin{abstract}
The treatment of information and digital competence is part of one of the competences that must be achieved in students, which consists of having a series of skills to search, obtain, process and communicate information, and include the new technologies of the information and communication, extracting its maximum performance from its understanding, and where the present research aims to analyze the use of technological tools in the achievement of mathematical competencies in virtual university education, using technological surveillance in the search for updated information, filtering in shorter periods, the use of Mendeley Desktop software as a sharing tool for analyzed documents; being the signing technique and syncretic files as information processing instruments, from which it is inferred that ICTs have allowed a substantial change in the education sector as a facilitator of knowledge with participatory methodologies, use of educational platforms integrating technological strategies innovative, which have managed to impact on the quality training of the student, changing their cultural and social behavior, achievements in rapid and timely research by strengthening their learning, search skills, communication channels, interactivity and saving time.
\end{abstract}

Keywords: competence; communication; information; mathematics; technologies.

Artículo recibido: 05 de Abril 2021 Aceptado para publicación: 28 de Mayo 2021 Correspondencia: junuche@ hotmail.com Conflictos de Interés: Ninguna que declarar 


\section{INTRODUCCIÓN}

En la educación, la aplicación de las Tecnologías de la Información y Comunicación (TICS) ha logrado un resultado efectivo con la enseñanza de los discentes en todos los niveles, además apoya en fortalecer las habilidades blandas en nuestra sociedad, pues las herramientas tecnológicas se desarrollan cada día más, como un soporte general en las personas, asimismo el uso de las TICS requiere de la asesoría de los docentes, a fin de aprovechar adecuadamente y alcanzar un aprendizaje continuo para toda la vida ( Vásquez, 2017).

Permite cambios en lo social y de manera pertinente en la educación, apoyando la construcción en el conocimiento, difusión de la información e interacción en todas las formas; siendo conveniente profundizar los programas e identificar como se está evolucionando las tecnologías desde una perspectiva pedagógica, a fin de estar debidamente actualizados y alcanzar de forma integral las competencias deseadas en los estudiantes ( Buitrago, 2018).

Para Escardíbul y Mediavilla (2016), con el uso de las TICS se confirma la existencia de efectos positivos con la competencia evaluada en matemáticas y comprensión lectora. Con los estudiantes habituados a las tecnologías, mejor dominio y adaptación, se logran mejores resultados en el aprendizaje de las matemáticas siendo herramientas que apoyan los procesos con aplicación de metodologías adecuadas y cambio de posturas en el estudiante.

La competencia matemática representa la habilidad para relacionar los números, solucionar problemas abstractos y servir como soporte a las demás ciencias, aplicando herramientas adecuadas para tomar decisiones de distinto grado de complejidad. Con la diversidad de herramientas que aportan las TICS, permiten despertar el interés y participación activa del estudiante que origina probablemente un rendimiento mejor, creatividad y espíritu de investigación.

En pleno siglo XXI se vislumbra el desarrollo científico-tecnológico en relación con la economía, cultura, medio ambiente y cambio permanente con las formas de aprender, enseñar y comunicar; como el aprendizaje de la matemática con la integración de las TICS donde el internet representa un papel preponderante en su aprendizaje, siendo su uso una necesidad en la sociedad actual y más utilizado de forma permanente en la creación e intercambio de información y del conocimiento (Rosero, 2018). 
La matemática constituye un apoyo para resolver problemas, desarrollo del pensamiento crítico, así como algoritmos que contribuyen en el desarrollo del perfil profesional. El beneficio se puede encontrar con el uso de software como el Winplot, Derive, Mathlab, Maple, GeoGebra con una aplicación en el álgebra, geometría y gráficos, cuya finalidad es realizar cálculos simbólicos y numéricos en diferentes modelos matemáticos.

El software en simulaciones resulta más eficaz en las matemáticas y demás ciencias, pues permite realizar simulacros de procesos antes de ocurrir los hechos de forma real, mientras que los procesadores de textos permiten crear, editar textos en una computadora con funcionalidades de redacción, tipografías diferentes y tamaños de letra, con aplicaciones gratuitas como: Openoffice, WPS Office, Draft, Libre Office y Google Docs. Soto (2018), afirma que las TICS son una ayuda para el docente en la enseñanza de sus clases, pues permiten la entrada a una vasta información y el uso de recursos que no se puede obtener de otra forma. El acercamiento a la información como audios, videos, imágenes, textos es de forma inmediata, permitiendo a los docentes reducir el tiempo y ampliar la flexibilidad de sus clases, así como la eficacia en el logro de actuales conocimientos con los estudiantes.

Con la aparición del Corona Virus SARS-CoV-2 el 2019 (COVID-19) a fines de noviembre en China y posteriormente en el mundo entero, trajo como consecuencias la paralización de los mecanismos del transporte, cierre de fronteras entre países, colapso en los hospitales por aumento del número de pacientes y la manera de enseñar en las instituciones educativas de presencial a una virtual, razón por la cual se justifica el presente estudio, a fin de evaluar los aspectos significativos originados en dicho cambio por ser excepcional.

Con la multiplicidad de herramientas pedagógicas que ofrecen las TICS, es necesario realizar la presente investigación cuyo objetivo general es: analizar las TICS en el logro de las competencias matemáticas en la educación virtual universitaria, siendo los objetivos específicos: a) describir las estrategias de aprendizaje con apoyo de las TICS en las competencias matemáticas, b) identificar las aplicaciones de las TICS que son necesarias implementar en la educación universitaria c) explicar la importancia del uso de entornos virtuales de aprendizaje de última generación. 


\section{METODOLOGÍA}

Un estudio científico de revisión de literatura es una investigación que analiza resultados científicos publicados en un campo del conocimiento, cuya finalidad es suministrar un escenario a la temática seleccionada, garantizando que la información es la más impactante de un conjunto de informes que por su naturaleza pueden ser extensos y de mucha antigüedad, y por lo tanto amerita una selección pre liminar como primer paso de la información a analizar (Gómez et al., 2014).

Una investigación científica es una condición deductiva que propone una estrategia metodológica en las dimensiones epistemológicas y pedagógicas para su análisis en evaluación del aprendizaje y formular una reflexión epistemológica sobre la construcción de un objeto de estudio sobre la esencia y límites del conocimiento del acto cognitivo, siendo una herramienta fundamental en las universidades para investigar y crear escenarios de investigación (Guevara, 2016).

En la investigación se utilizó la vigilancia tecnológica como un proceso organizado, permanente y selectivo en la búsqueda de información relevante y actualizada: Scopus, Ebsco, tesis de maestría y doctorales, revistas indizadas pues constituyen una fuente de ideas de actualidad, aplicando criterios de inclusión y exclusión en razón a la infinidad de información se procedió a filtrar la información en periodos más cortos.

Tabla 1. Ecuaciones de búsqueda utilizadas en Scopus, Ebsco

\begin{tabular}{lcc}
\multicolumn{1}{c}{ Ecuación de Búsqueda } & Resultados & Periodo \\
\hline TIC & 589 & $2016-2020$ \\
TIC + Competencias en matemáticas & 663 & $2018-2020$ \\
Uso de plataformas en matemáticas universitarias & 597 & $2018-2020$ \\
\hline
\end{tabular}

Fuente: Base de datos de Scopus y Ebsco

En la sistematización de la información se ha utilizado el Mendeley DesKtop, en la importación, exportación y compartimiento de documentos analizados en el presente trabajo de investigación. Los software son herramientas utilizadas en la organización de los procesos de las instituciones con eficiencia y satisfacción en función de sus reales necesidades, razón por la cual deben tener los parámetros que garanticen una calidad con mejora continua y de última generación (Callejas et al., 2017). 
La sistematización tiene gran relevancia para conocer las etapas llevados a cabo y sobre todo para tomar como referencia ese importante acervo de investigación que se ha generado a través de investigadores en publicaciones diversas en el tiempo. Proporcionar la metodología e instrumento prosiguiendo la sistemática informativa, señalándose las siguientes etapas: a) Identificar fuentes de información b) Recopilar información c) Ordenar y clasificar datos estructurados bajo la forma de categorías, en una base de datos organizadas d) Analizar e) Presentación.

Como técnica se ha utilizado el fichaje, definida como un modo de recopilar y almacenar información, donde cada archivo contiene una serie de datos de extensión variable, pero referida a un mismo tema y la ficha sincrética utilizada como instrumento de procesamiento y ordenamiento de los datos en la investigación. Existe una variedad de tipos de fichas utilizados especialmente por los investigadores: Textuales, resúmenes, comentarios y paráfrasis, donde cada una de ellas tiene una característica especial de diseño. La ficha sincrética utilizada para tal fin contiene seis secciones distribuidas en: Título de la investigación, referencia bibliográfica donde se indica el autor o autores, así como la página de consulta, el contenido que ha servido para formular la presente investigación, abreviatura de la ficha, ubicación de la página en el trabajo y número de página donde se ubica la referencia bibliográfica.

\section{Modelo de ficha sincrética}

\begin{tabular}{|c|c|c|}
\hline \multirow[t]{2}{*}{$\begin{array}{l}\text { Ubicación } \\
\text { en el } \\
\text { Trabajo }\end{array}$} & $\begin{array}{l}\text { Datos del trabajo científico } \\
\text { Título: } \\
\text { Autor: }\end{array}$ & \multirow[t]{2}{*}{$\begin{array}{l}\text { Tipo de } \\
\text { ficha } \\
\text { sincrética }\end{array}$} \\
\hline & Fuente de referencia & \\
\hline $\begin{array}{l}\text { No de } \\
\text { página del } \\
\text { trabajo }\end{array}$ & Título y contenido de la ficha sincrética & \\
\hline
\end{tabular}




\section{RESULTADOS}

Con la creciente incorporación de las TICS en la educación, que abarca motores de búsqueda de recursos compartidos ayudando a pensar y comunicarse creativamente, aparecen nuevas formas de aprendizaje mediante la utilización de herramientas con un aprendizaje más significativo, siendo el estudiante protagonista del aprendizaje y de su conocimiento logrando un cambio de paradigma en el sector educativo, que facilita la gestión del conocimiento y un avance en el futuro de las universidades, la autorregulación del aprendizaje ocupa en la investigación un espacio privilegiado, puesto que los estudiantes intervienen de manera activa con el fin de alcanzar sus objetivos (Petruo et al., 2020).

Los estudios requieren la promoción de mejoras en la calidad educativa, en donde el docente desempeña un papel influyente en el desarrollo del currículo y por consiguiente, guía en el proceso del aprendizaje asociado en el rendimiento académico con metodologías activas y participativas en los modelos educativos, incluyéndose la educación virtual a través de plataformas con recursos didácticos para un aprendizaje independiente mediadas por las TICS y superar la presencia física en épocas de pandemia, donde los medios tecnológicos proponen nuevas estrategias metodológicas (Mendoza et al., 2019).

Las prácticas en matemáticas con apoyo de las TICS, son el reflejo del nivel de competencia que deben tener los docentes aunados al conocimiento tecnológico, contribuyendo al sistema educativo con calidad y formación integral del educando donde las tecnologías son estructuradas en función del aprendizaje y será más eficaz de acuerdo al dominio que tengan los docentes sobre estos conocimientos superando los niveles tradicionales en los constructos matemáticos e innovadores en las prácticas pedagógicas (Arévalo et al., 2019).

La formación de docentes en herramientas tecnológicas, significa el análisis de sus dimensiones y principios en diferentes estudios y trabajos, quedando claro su importancia en mejorar la calidad, el desempeño educativo y las transformaciones que resultan durante su aplicación, se analiza la formación profesional en las competencias digitales que deben poseer los docentes en su incorporación y práctica profesional, propiciando nuevas formas de interacción entre estudiantes y docentes; favoreciendo el acercamiento a los contenidos y el desarrollo de las inteligencias múltiples del estudiante, creando ambientes 
flexibles y enriquecidos de aprendizaje con presencia de tecnologías emergentes (Almenara \& Gimeno, 2019).

En cuanto a la influencia de las TICS y el impacto que ha generado en la enseñanza universitaria de Colombia, los resultados conducen a importantes aportes en el contexto educativo como fenómeno de alta incidencia de promover la innovación como mecanismo de competencia con aplicaciones cada vez mayores, donde el sistema intenta adaptarse a estos cambios a través de políticas educativas que integren estas herramientas en el aprendizaje (De La Hoz et al., 2018).

Al analizar la dinámica de las universidades de Barranquilla en Colombia, con respecto a la integración de las TICS y su innovación en la docencia universitaria, se evidenció que el $71 \%$ de las universidades no presentan capacitación en tecnologías actualizadas y además el $64 \%$ con un diseño de metodologías mediadas por TICS con participación de docentes. Por tanto, es necesario formular estrategias de articulación con tecnologías innovadoras para fortalecer la docencia, teniendo en cuenta que se han desarrollado cambios significativos en los patrones educativos y modelos de aprendizaje basados en competencias, para formar profesionales y actuar de forma dinámica en el actual mundo globalizado (Marín et al., 2017).

Las TICS han cambiado las formas de comportamiento, pues son parte del entorno familiar, social y educativo, donde las generaciones nativas de la era digital han sido testigos de su evolución y se relacionan con otras generaciones que han visto la innovación tecnológica sin utilizar las tecnologías; como son los docentes que se resisten a este vertiginoso cambio por la forma tradicional de enseñar. En consecuencia, es conveniente realizar cambios en la educación para que los estudiantes y profesores se adapten de forma inmediata, buscando nuevas opciones didácticas en paralelo al uso de las tecnologías educativas, teniendo en cuenta factores sociales, demográficos y económicos (Rodríguez et al., 2018).

Se relaciona los roles directos de las tecnologías de la información y comunicación con una sociedad moderna, reconociéndola como uno de los principales recursos que generan capacidad de producción y un aumento en el ritmo de trabajo con una generación de impacto en la vida, donde se automatiza y robotiza las ramas de producción, creando un sistema de integración de la comunicación, mejorando las fuentes y portadores de información con una transformación radical permanente ( Isachenko, 2018). 
La incorporación de las tecnologías educativas deben integrarse en la formación pedagógica en las instituciones universitarias mexicanas, tomando en cuenta la percepción de los docentes adscritos a carreras profesionales en ciencias administrativas públicas, con altos niveles de intención de uso adecuado, pero con baja frecuencia; por otro lado, existe una centralidad en la adquisición de equipos tecnológicos fijos y de uso personal, un aumento en la adquisición de infraestructuras y una disminución de gasto en la formación docente. La implantación de las tecnologías a nivel universitario es un gran desafío en la formación docente, con ausencia de preparación en este ámbito, incluida la permanencia de los docentes con 45 años de servicio, siendo el sector que menos utiliza las TICS, por lo que es conveniente incluir nuevas políticas educativas y perfeccionar la enseñanza en el nivel universitario (Zempoalteca et al., 2018).

La gestión del conocimiento, se puede precisar como una transformación sistemática para adquirir, organizar y comunicar el conocimiento de los recursos humanos para ser más efectivo, donde las universidades son las principales administradoras de la gestión del conocimiento, apoyando con diversidad de estrategias y capitalización del conocimiento. Paralelamente, las aulas virtuales constituyen un recurso tecnológico como nuevo soporte en los procesos del conocimiento, apoyando la educación presencial o Blended-learning (Mariño, 2018).

La propuesta de una investigación, cuyo objetivo fue establecer cómo los docentes promueven el uso competencial transversal de las TICS en los nuevos docentes y que a través del análisis realizado con el programa Atlas. ti, se encontró que incentivan a los estudiantes a utilizar dichas tecnologías como recurso didáctico, dinamizar las clases con promoción de la interacción, uso de internet, desarrollo de redes de aprendizaje demostrando logros en el desarrollo de su trabajo y uso de software para aprender matemáticas ( Varela et al., 2020).

El principal reto en la educación para la evaluación de competencia en matemáticas universitarias, se considera a la rúbrica como la herramienta más conveniente en realizar una evaluación estableciendo los criterios de evaluación con sus indicadores de logro en contenidos, habilidades blandas y duras de carácter profesional, actitudes y estrategias de aprendizaje utilizando como medio las TICS (Masero et al., 2018). Una evaluación por competencia debe medir la capacidad del estudiante que tiene al enfrentar realidades y soluciones a diversidad de problemas para toda la vida, donde las TICS en las 
universidades, se convierten en aliados como estrategias valiosas para lograr progresivamente superar los obstáculos en los procesos de aprendizaje.

La aplicación de las matemáticas a situaciones reales del mundo es una de las principales funciones en la formación de ingenieros, por lo que es necesario identificar las competencias matemáticas en la resolución de problemas, siendo una preocupación de la sociedad actual por los resultados obtenidos desde una perspectiva de calidad, donde en un nivel superior presentan dificultades en el aprendizaje de las matemáticas universitarias, y el objeto límite representa el primer obstáculo para los estudiantes de ingeniería (Díaz \& Poblete, 2019).

Las tecnologías han logrado un cambio en el sector educativo, al incorporarlas en el intercambio y apoyo en el conocimiento desde su aparición y evolución de la informática, donde al hacer uso de objetos virtuales de aprendizaje como recursos digitales estructurados de forma significativa, auto contenible y reutilizable, cambian la concepción mecánica de la asignatura del cálculo diferencial al efectuar cálculos en situaciones propias de su campo de estudio al aumentar el tiempo en su reforzamiento (Martínez et al., 2018). Se puede mencionar el uso del software AutoCAD Civil 3D, que permite entregar proyectos de ingeniería y urbanismo en menor tiempo, de mayor calidad y mantener datos más coherentes.

En la investigación sobre la apropiación de las herramientas tecnológicas entre jóvenes de clases populares argentinas, como teléfonos móviles, computadoras e internet, se infiere que los modos de ser jóvenes en nuestra sociedad contemporánea constituyen nuevas formas habilitadas a la hora de gestionar el tiempo, organizar el espacio, además de articular la privacidad, intimidad y los problemas públicos, impulsados por las tecnologías. Estos patrones no son homogéneos; por el contrario, adquieren significados diferentes según el contexto de apropiación sociocultural y la forma de experimentar el tiempo y el espacio son trascendentales en una revolución científico-tecnológica sin precedentes (Benitez, 2018).

El futuro de la educación virtual a distancia, está sujeto a una transformación constante de las tecnologías digitales y la innovación de las instituciones con nuevas rutas metodológicas en diferentes áreas del conocimiento y con la creciente creación de programas y cursos de educación superior que actualmente se están desarrollando a 
distancia, se proyecta un futuro favorable, para responder a la necesidad de brindar educación a una población de diferentes regiones (Sucerquia et al., 2016).

De acuerdo con los resultados de la investigación en el uso de herramientas tecnológicas, para identificar factores que habilitan el trabajo colaborativo, el análisis crítico y el autoaprendizaje, los estudiantes asumen roles con mayor compromiso en su aprendizaje donde la transformación de la World Wide Web cambió sitios y páginas web, navegadores y herramientas multimediales, que facilitan múltiples espacios virtuales, siendo factores importantes en los entornos educativos (Rugeles et al., 2015).

Las tecnologías han cambiado aspectos de la vida y en la educación el efecto de las herramientas pedagógicas son elementos indiscutibles de su presencia para el desarrollo pedagógico en la enseñanza, donde los docentes con mayor dominio son quienes manejan mejor su uso didáctico, la creación de plataformas educativas y el uso generalizado de dispositivos móviles ha enriquecido la interacción de forma inmediata y eficaz entre estudiantes y docentes, más aún con aquellos estudiantes nativos digitales ( Prada et al., 2019).

La incorporación de la tecnología ha permitido la integración de conceptos como el aprendizaje móvil y las plataformas virtuales, que favorecen la actualización de conocimientos, y al analizar el impacto en el rendimiento académico, mediante el uso del video como recurso en la modalidad Blended learning de la asignatura de matemáticas, se detectó una mejora en el rendimiento académico del grupo comparativo de experimentación al de los sujetos a control, con alto grado de satisfacción y motivación a la modalidad de estudio a distancia con el uso de video ( Rodríguez et al., 2017).

Las matemáticas siempre han generado controversias en su enseñanza porque se obtienen bajos rendimientos y paralelamente como ciencia fundamental al ser soporte a las demás ciencias, con la creencia que para su entendimiento y comprensión se requiere una inteligencia superior, lo cual gesta una inseguridad y desmotivación en el aprendizaje de las matemáticas, como sucede en distintos niveles de la educación; sin embargo sus procesos de enseñanza deben ser analizados desde varias aristas como son: factores sociales, educativos y personales, que deben ser evaluados oportunamente (González, 2019).

Conforme a Grisales (2018), la enseñanza de la matemática con uso de recursos tecnológicos, origina un logro positivo en los estudiantes, sin embargo es conveniente 
efectuar otros estudios en tiempo más amplios, además se requiere articular las competencias comunicativas y tecnológicas con los currículos de formación de los estudiantes y por consiguiente en los docentes, pues deben cambiar los métodos tradicionales de enseñanza de esta área, carentes de un rendimiento adecuado en los estudiantes.

Durante los últimos años se ha visto una constante integración de las tecnologías, por lo que es necesario incluir las tecnologías con las variables tradicionales del rendimiento académico especialmente aquellas con el entorno tecnológico de la institución, accesibilidad y uso de internet por tener una incidencia significativa en distintos niveles en el trabajo del estudiante, como la búsqueda de información seleccionando fuentes, organizando e integrando la información útil en la resolución de problemas (García \& Cantón, 2019).

El desarrollo de la electrónica ha permitido el perfeccionamiento de las tecnologías, pero su implantación en las instituciones educativas no es suficiente, siendo necesario realizar cambios en la forma de enseñar, sus procedimientos, modificación de los contenidos y logros en la formación de los estudiantes, puesto que el proceso de enseñanza en matemáticas no ha permitido resultados ventajoso en estudiantes universitarios debido a las dificultades que se muestran en la apropiación del conocimiento de las matemáticas, siendo necesaria la integración de manera eficiente (Olivier, 2016). La tecnología ha tenido un resultado sociocultural ya que permite cambiar hábitos en la forma de comunicarse y trabajar, así como los conceptos y principios matemáticos se incrementan por el binomio tecnología vs matemática, siendo un requisito indispensable la integración de las tecnologías a través del uso de la plataforma Moodle, con estrategias adecuadas y donde los docentes tutores analicen los recursos tecnológicos de manera efectiva y oportuna (Sánchez, 2015).

La incorporación de sistemas algebraicos computacionales (SAC) a dispositivos móviles de estudiantes de ingeniería en la asignatura de ecuaciones diferenciales para su adquisición de capacidades y habilidades en su resolución así como la gráfica de resolución, el software EDO del Tecnológico Nacional de México, sugiere el uso de TICS, para obtener competencias en la resolución de gráficos, donde el software se registra con ACF-0905 (Camacho et al., 2019). 
Una investigación sobre el modelado de las matemáticas con articulación al uso de software en el proceso de educación superior, se habilita como alternativa pedagógica en situaciones reales, así como de interés en los estudiantes basada en el uso de la computadora, promoviendo un ambiente propicio al desarrollo del conocimiento de las matemáticas, aportando creatividad y autonomía en el desarrollo de las actividades del resultado y rendimiento académico (De Carvalho et al., 2019).

Según Gómez et al. (2020), el aprendizaje-servicio es una metodología que está adquiriendo mayor alcance a nivel universitario, vinculando el aprendizaje del estudiante con el servicio a la comunidad, alcanzando logros en el currículo académico, formación en valores y responsabilidad social, cuya implementación en proyectos para el área de las matemáticas brinden una herramienta didáctica que examine cómo el aprendizajeservicio fortalece las competencias específicas y transversales, y que en el contexto actual responda a aprender, hacer, vivir y de ser con una educación de calidad.

La formación profesional conduce a la mejora de la calidad a través de la integración de nuevas tecnologías entre docentes y alumnos, como laboratorios virtuales, Aprendizaje Basado en Problemas y aplicación de las TICS, generando aprendizajes significativos con resultados académicos positivos, no basta con aplicar metodologías didácticas, sino la verificación de lo aprendido en su formación universitaria, siendo necesario proponer nuevos roles constructivistas en la docencia y superar el cambio de la forma tradicional de conducir la enseñanza-aprendizaje (Faúndez et al., 2017).

El Ministerio de Educación Nacional de Colombia, aboga por el uso de tecnologías y su influencia en las prácticas pedagógicas, investigando a grupos de docentes sobre el conocimiento de las tecnologías, encontrándose limitaciones en el conocimiento de los recursos tecnológicos en los procesos de las matemáticas y el fracaso con las prácticas pedagógicas de esta competencia y que en los programas no se integra adecuadamente en su preparación profesional, siendo su relevancia significativa cuando se capacita adecuadamente al docente (Padilla y Conde, 2020).

Una plataforma educativa virtual, son programas que habilitan ambientes virtuales, su aplicación se sintoniza debido a la pandemia, donde la tecnología simula la educación presencial, resaltando que diversas instituciones educativas vienen utilizando aulas virtuales con creación de espacios de interacción, evaluación del estudiante, donde la educación en línea está creciendo considerablemente, eliminando barreras de distancia y 
tiempo, articulando diferentes recursos multimedia, videos, chats, videoconferencias, calificación de tareas, exámenes en línea (Tobón et al., 2018).

Los nuevos desafíos que han surgido con la era de la alfabetización digital en la educación han permitido el diseño de plataformas virtuales como: Schoology, Edmodo, Chamilo, Canvas LMS y Moodle, ideales para la creación de cursos y entornos de aprendizaje personalizados, las cuales permiten una excelente comunicación entre estudiantes y docentes, evaluaciones en línea, chats, videoconferencias, foros y fortalecimiento de aprendizajes por ser un repositorio de contenidos, donde muchas instituciones educativas no contaban con estos recursos con la aparición del COVID-19.

No hay duda del beneficio que proporcionan las tecnologías de la información y comunicación, surgiendo la interrogante sobre la responsabilidad de enseñar considerando el impacto de los residuos tecnológicos en el mundo, los mismos que deben incluirse en los programas educativos, pues la fabricación de dispositivos tecnológicos provoca una actividad con impacto ambiental, asimismo las industrias programan una vida útil de la tecnología para que los usuarios recambien por unas nuevas, pero de forma excepcional el COVID-19 ha provocado la adaptación digital, incrementando el uso de tecnologías como alternativa a la actividad presencial (Barragán et al., 2020).

\section{CONCLUSIONES}

El beneficio de usar plataformas en la educación virtual, permite que el docente pueda grabar sus clases como videoconferencias con antelación mediante el Zoom y los estudiantes a través de los enlaces de las videoconferencias grabadas los pueden usar cuantas veces deseen, incluyendo a aquellos estudiantes que no puedan asistir a las clases virtuales.

1) Las Tecnologías de la Información y Comunicación conforme han evolucionado, han tomado posición en el sistema educativo para lograr una formación de calidad en el estudiante, configurándose como un instrumento vital en las actividades educativas. Desde sus antecedentes, la informática se resume desde el punto de vista del software, internet y de los videos juegos; siendo los ordenadores la revolución que han ocasionado dentro de las comunicaciones y redes, destacándose como la era informática como consecuencia del uso del internet.

2) La red de redes ha permitido que la comunicación unidireccional de un emisor alcance a miles de usuarios oyentes o lectores, donde las nuevas generaciones se han visto 
influenciadas por el uso de las tecnologías educativas, por lo que su comportamiento cultural y social es diferente de aquellas que no han logrado integrarse al uso, por diversas razones como su ubicación geográfica, edad, económicas y sociales.

3) Las tecnologías en paralelo con el internet como herramientas en el ámbito educativo, está permitiendo logros significados en cuanto al aprendizaje e investigación de forma rápida y oportuna, los cuales se pueden analizar desde sus diferentes dimensiones. Las matemáticas constituyen un apoyo para resolver problemas, desarrollo del pensamiento crítico, así como algoritmos que contribuyen en el desarrollo de su perfil profesional.

4) Las herramientas educativas con uso alterno de plataformas virtuales y soporte tecnológico, conduce a que el aprendizaje se reduzca el espacio físico a la digital y con una visión del aprendizaje netamente virtual del futuro, donde el rendimiento académico, refleja las capacidades adquiridas de los estudiantes en relación a lo aprendido durante un proceso formativo, respondiendo además a los incentivos educativos y aptitud propia de cada estudiante.

5) El mayor efecto que proporcionan las TICS, es el aumento de habilidades de búsqueda, acceso flexible en la información, mayores canales de comunicación, interactividad, reducción de costos, ahorro de tiempo y energía en la realización de trabajos por parte de los estudiantes y docentes. La educación virtual con aplicaciones tecnológicas aún empieza y será una educación sin barreras físicas donde el estudiante asumirá nuevos roles como principal actor de su autoaprendizaje.

\section{RECOMENDACIONES}

El diseño y programación de software en cada ciclo del estudiante universitario, en concordancia con la malla curricular de cada programa de estudio, incluyendo algunos de carácter obligatorio como el SPSS que contiene diversas funciones estadísticas y del turnitin, a fin de ir capacitando a los estudiantes progresivamente en la elaboración de su tesis.

1) Se requiere que los docentes y estudiantes tengan una formación y capacitación permanente en las aplicaciones de las herramientas tecnológicas, pues los escenarios educativos han evolucionado desde la modalidad presencial, semipresencial y virtual, teniendo la necesidad de determinar cuáles son las que influyen en el logro de un mejor rendimiento académico. 
2) La implantación de plataformas proporciona una gama de ventajas en estudiantes y docentes que se apoyan en el trabajo habitual, siendo su aplicación un requisito indispensable en las instituciones educativas, por lo que es conveniente mejorar los presupuestos asignados en la educación. A excepción de Cuba que asigna un porcentaje del gasto público con dos cifras, en los demás países de América Latina alcanza un promedio del 5,4\% PBI.

3) La educación debe centrarse en las tres $\mathrm{C}$ : curiosidad, pensamiento crítico y creatividad, centrarse en el trabajo en equipo, comunicación y colaboración, para formar humanos creativos y empáticos, donde los docentes deben ser líderes de la sociedad que marcan la vida en los estudiantes, integrando la inteligencia artificial en las aulas y el desarrollo de las habilidades blandas requeridas por el mercado laboral.

4) Con el ritmo creciente de la tecnología los dispositivos que se usan se renuevan constantemente, desechando a diario toneladas de productos electrónicos, estimándose alrededor de 50 millones de tonelada anuales en desechos electrónicos con presencia de metales pesados como el mercurio y plomo de impacto en el medio ambiente, por lo que debe adoptarse políticas de renovación de equipos electrónicos biodegradables.

5) Formalizar un nuevo sistema educativo, para formar ciudadanos del mundo con respeto a las leyes, la ética, ecología, el medio ambiente, integrándose el uso de herramientas tecnológicas en todos los niveles educativos, con una visión hacia una enseñanza virtual en el futuro.

\section{REFERENCIAS BIBLIOGRÁFICAS}

Almenara, J. C., \& Gimeno, A. M. (2019). Information and Communication Technologies and initial teacher training. Digital models and competences. Profesorado, 23(3), 247-268.

https://doi.org/10.30827/profesorado.v23i3.9421

http://revistaseug.ugr.es/index.php/profesorado/article/view/9421/9308

Arévalo, M.A., García, M.A., y Hernández, C.A. (2019). Competencias TIC de los docentes de matemáticas en el marco del modelo TPACK: Valoración desde la perspectiva de los estudiantes. Civilizar, 19(36), 115-132.

https://doi.org/10.22518/usergioa/jour/ccsh/2019.1/a07

https://revistas.usergioarboleda.edu.co/index.php/ccsh/article/view/v19n36a07 
De Carvalho, S., Da Silva, R., y Basniak, M.I. (2019). Modelización en la enseñanza de matemáticas: un análisis de las contribuciones de software en la enseñanza superior. Paradigma, ISSN 1011-2251, Nº. 1, 2018, págs. 282-300

https://dialnet.unirioja.es/servlet/articulo?codigo=7304252

Barragán, R., Corujo, M. C., Palacios, A., y Román, P. (2020). Teaching Digital Competence and Eco-Responsible Use of Technologies: Development and Validation of a Scale. Sustainability, 12(18), 7721.

https://doi.org/10.3390/su12187721

https://www.mdpi.com/2071-1050/12/18/7721

Benitez, S., (2018). La experiencia juvenil del tiempo y el espacio a partir de la apropiación de las Tecnologías de Información y Comunicación en La Plata, Argentina. Andamios, 15(36), 343-368. https://doi.org/10.29092/uacm.v15i36.613

http://www.scielo.org.mx/scielo.php?script=sci_abstract\&pid=S1870-

$00632018000100343 \& \operatorname{lng}=\mathrm{es} \& n \mathrm{~nm}=$ iso

Buitrago, A. M. (2018). Presencia de las tecnologías de la información y la comunicación (TIC) en los programas iniciales de formación de profesores de matemáticas en las últimas tres décadas [Tesis de maestría, Universidad Pedagógica Nacional, Colombia]. Repositorio Institucional. http://repositorio.pedagogica.edu.co/bitstream/handle/20.500.12209/11457/TE22402.pdf? sequence $=1 \&$ isAllowed $=\mathrm{y}$

Callejas, M., y Alarcón, A.C. (2017). Modelos de calidad del software, un estado del arte. 13(1).

https://doi.org/10.18041/entramado.2017v13n1.25125

https://revistas.unilibre.edu.co/index.php/entramado/article/view/428

Camacho, A., y Caldera, M.I., y Valenzuela, V. (2019). Fidelity in the use of app for solving differential equations. Apertura, vol. 11, (1) Abril-September.

https://doi.org/10.32870/Ap.v11n1.1463

http://www.udgvirtual.udg.mx/apertura/index.php/apertura/article/view/1463/0

De la Hoz, E., Martínez, O., Combita, H., y Hernández, H. (2018). Las Tecnologías de la Información y la Comunicación y su Influencia en la Transformación de la Educación Superior en Colombia para Impulso de la Economía Global. № 1-2019 Recibido Abr. Información Tecnológica, 30(1), 255-262. 
https://doi.org/10.4067/S0718-07642019000100255

https://scielo.conicyt.cl/scielo.php?script=sci_arttext\&pid=S0718-

07642019000100255\&lng=en\&nrm=iso\&tlng=en

Díaz, V., \& Poblete, Á. (2019). Competencias matemáticas: desempeño y errores en la resolución de problemas de límites. Paradigma, 40(1), 358-383.

https://doi.org/10.37618/PARADIGMA.1011-2251.2019.p358 - 383.id733

http://revistaparadigma.online/ojs/index.php/paradigma/article/view/733

Escardíbul, J. O., y Mediavilla, M. (2016). El efecto de las TIC en la adquisición de competencias. Un análisis por tipo de centro educativo. Revista española de pedagogía, 317-335.

https://reunir.unir.net/bitstream/handle/123456789/5059/El-efecto-de-las-

TIC.pdf? sequence $=1$

Faúndez, C. A., y Bravo, A.A., Ramírez, G. P., y Astudillo, H.F. (2017). Tecnologías de la Información y la Comunicación (TIC) en el Proceso de Enseñanza-Aprendizaje de Conceptos de Termodinámica como Herramienta para Futuros Docentes. Formación Universitaria, 10(4), 43-54. https://doi.org/10.4067/S0718-50062017000400005 https://scielo.conicyt.cl/scielo.php?script=sci_arttext\&pid=S0718$50062017000400005 \& \operatorname{lng}=$ en\&nrm=iso\&tlng=en

García, S., \& Cantón, I. (2019). Use of technologies and academic performance in adolescent students. [Uso de tecnologías y rendimiento académico en estudiantes adolescentes]. Comunicar, 59, 73-81.

https://doi.org/10.3916/C59-2019-07

https://www.revistacomunicar.com/index.php?contenido=detalles\&numero=59\&art iculo $=59-2019-07$

Gómez, E., Fernando, D., Aponte, G., y Betancourt, L.A. (2014). Literature review methodology for scientific and information management, through its structuring and systematization Metodología para la revisión bibliográfica y la gestión de información de temas científicos, a través de su estructuración y sistematización. DYNA, 81(184), 158-163.

https://www.redalyc.org/pdf/496/49630405022.pdf 
Gómez, I. M., y Ortuño, T., y De la Fuente, A. (2020). Aprendizaje-Servicio en Matemáticas: Uso de Trayectorias de Aprendizaje en la formación universitaria. REDU. Revista de Docencia Universitaria, 18(1). pp. 213-231.

https://doi.org/10.4995/redu.2020.12079

https://polipapers.upv.es/index.php/REDU/article/view/12079/12785

González, L. (2019). The Virtual Classroom as a Tool for Increasing Satisfaction Levels in the Study of Mathematics. Information Tecnológica, 30(1), 203-214. https://doi.org/10.4067/S0718-07642019000100203

https://scielo.conicyt.cl/scielo.php?script=sci_arttext\&pid=S0718-

07642019000100203\&lng=en\&nrm=iso\&tlng=en

Grisales, A. (2018). Uso de recursos TIC en la enseñanza de las matemáticas: retos y perspectivas. Entramado. 14(2): 198-214.

https://doi.org/10.18041/1900-3803/entramado.2.4751

http://www.scielo.org.co/scielo.php?script=sci_arttext\&pid=S190038032018000200198

Guevara, R. (2016). El estado del arte en la investigación: ¿Análisis de los conocimientos acumulados o indagación por nuevos sentidos? Revista Folios, (44), 165-179. http://www.redalyc.org/articulo.oa?id=345945922011

Isachenko, N., (2018). The Role of Information and Informational and Communication Technologies in Modern Society. Utopía y Praxis Latinoamericana. No 82. pp. 361367. Revista Internacional de Filosofía y Teoría Social. https://doi.org/10.5281/zenodo.1512122 https://zenodo.org/record/1512122\#.YFO9Aq9KjIU

Marín, F.V., Inciarte, A., Hernández, H., \& Remedios, P. (2017). Strategies of Institutions of Higher Education for the Integration of Information and Communication Technologies and of Innovation in the Teaching Process. A Study in the District of Barranquilla, Colombia. Formación universitaria, 10(6), 29-38

https://doi.org/10.4067/S0718-50062017000600004

https://scielo.conicyt.cl/scielo.php?script=sci_arttext\&pid=S0718-

$50062017000600004 \& \operatorname{lng}=\mathrm{en} \& \mathrm{nrm}=\mathrm{iso} \& \operatorname{tn} \mathrm{ln}=\mathrm{en}$ 
Mariño, S. I. (2018). Tecnologías de la información y comunicación (TIC) para el apoyo de procesos de gestión del conocimiento en aulas virtuales. Revista Educación en Ingeniería, 13(26),-81.

https://doi.org/10.26507/rei.v13n26.919

https://www.educacioneningenieria.org/index.php/edi/article/view/919

Martínez, O., Combita, H., y De la Hoz, E. (2018). Mediation of Virtual Learning Objects in the Development of Mathematical Competences in Engineering Students. Formación Universitaria, 11(6), 63-74.

https://doi.org/10.4067/S0718-50062018000600063

https://scielo.conicyt.cl/scielo.php?script=sci_arttext\&pid=S0718-

$50062018000600063 \& \operatorname{lng}=$ en\&nrm=iso\&tlng=en

Masero, I. C., Camacho, M.E., y Vázquez, M.J. (2018). Cómo evaluar conocimientos y competencias en la resolución matemática de problemas en el contexto económico a través de rúbricas. Revista Electrónica Interuniversitaria de Formación del Profesorado, 21(1), 51.

https://doi.org/10.6018/reifop.21.1.277981

https://revistas.um.es/reifop/article/view/277981

Mendoza, H. H., Burbano, V.M., \& Valdiviseo, M.A. (2019). The role of the teacher of mathematics in virtual university education. A study in the Pedagogic and Technologic University of Colombia. Formacion Universitaria, 12(5), 51-60.

https://doi.org/10.4067/S0718-50062019000500051

https://scielo.conicyt.cl/scielo.php?script=sci_arttext\&pid=S0718-

$50062019000500051 \& \operatorname{lng}=\mathrm{en} \& n r m=\mathrm{iso} \& \operatorname{lng}=\mathrm{en}$

Olivier, O. Z. (2016). El uso de las Tecnologías en la enseñanza-aprendizaje de la matemática en la Universidad Experimental de las Fuerzas Armadas, Núcleo Sucre. Santiago, Vol. (1). pp. 42-53.

https://santiago.uo.edu.cu/index.php/stgo/article/view/145160104/641

Padilla Escorcia, I. A. y Conde, R. J. (2020). Uso y formación en TIC en profesores de matemáticas: un análisis cualitativo. Revista Virtual Universidad Católica del Norte, (60), 116-136.

https://www.doi.org/10.35575/rvucn.n60a7

file://C:/Users/Ing\%20Cheng/Downloads/1166-4794-1-PB.pdf

Ciencia Latina Revista Científica Multidisciplinar, Ciudad de México, México. 
Petruo, V., Bodmer, B., Bluschke, A., Münchau, A., Roessne, V., \& Beste, C. (2020). Comprehensive Behavioral Intervention for Tics reduces perception-action binding during inhibitory control in Gilles de la Tourette syndrome. Scientific Reports, 10, 1174 .

https://doi.org/10.1038/s41598-020-58269-z

https://www.nature.com/articles/s41598-020-58269-z

Prada, R., Hernández, C.A., y Aloiso, A. (2019). Usos y efectos de la implementación de una plataforma digital en el proceso de enseñanza de futuros docentes en matemáticas. Revista Virtual Universidad Católica del Norte, (57), 137-156. https://doi.org/10.35575/rvucn.n57a10

https://revistavirtual.ucn.edu.co/index.php/RevistaUCN/article/view/1059

Rodríguez, D. G. L., Álvarez, G.V., López, S.H., \& Meza, M.E.C. (2018). Design of a PEAT (Teaching Learning Process with Technology) methodology to integrate Information and Communication Technology into Education. Proceedings of the LACCEI international Multi-conference for Engineering, Education and Technology, 2018-July, 2018, 19-21. https://www.scopus.com/inward/record.uri?eid=2-s2.0-

85057438466\&doi=10.18687\%2FLACCEI2018.1.1.119\&partnerID=40\&md5=fd3 4175c020d73dcae4bb87014fddd0c

Rodríguez, R. A., López, V.S., y Mortera, F.J. (2017). El video como Recurso Educativo Abierto y la enseñanza de Matemáticas. Revista Electrónica de Investigación Educativa, 19(3), 92-100. https://doi.org/10.24320/redie.2017.19.3.936 http://www.scielo.org.mx/scielo.php?script=sci_abstract\&pid=S1607$40412017000300092 \& \operatorname{lng}=\mathrm{es} \& n r m=$ iso

Rosero, J. R. (2018). Impacto del uso de las TIC como herramientas para el aprendizaje de la matemática de los estudiantes de educación media. Revista de la Facultad de Ciencias Médicas (Quito), 1(1), 70-91.

https://doi.org/10.29166/catedra.v1i1.764

https://revistadigital.uce.edu.ec/index.php/CATEDRA/article/view/764

Rugeles, P. A., Mora, B., y Metaute, P.M. (2015). El rol del estudiante en los ambientes educativos mediados por las TIC. Revista Lasallista de Investigación, 12(2), 132138. https://doi.org/10.22507/rli.v12n2a14

Ciencia Latina Revista Científica Multidisciplinar, Ciudad de México, México. ISSN 2707-2207 / ISSN 2707-2215 (en línea), mayo-junio, 2021, Volumen 5, Número 3. https://doi.org/10.37811/cl rcm.v5i3.497 p. 2928 
http://repository.lasallista.edu.co:8080/ojs/index.php/rldi/article/view/872

Sánchez, A. (2015). Estrategias para el aprendizaje de las funciones reales con la plataforma moodle. Enl@ce: Revista Venezolana de Información, Tecnología y Conocimiento, vol. 12, (2), pp. 41-54.

http://www.redalyc.org/articulo.oa?id=82340995004

https://www.redalyc.org/pdf/823/82340995004.pdf

Soto, E. (2018). Aplicación de las TICs para el Desarrollo de Habilidades Matemáticas Curriculares y del Aprendizaje Cooperativo de Estudiantes del Segundo Bachillerato. Machala. Ecuador.

http://repositorio.utmachala.edu.ec/bitstream/48000/12451/1/TTUACS-2018-DOI-

DE00002.pdf

Sucerquia, E.A., Londoño, R.A., Jaramillo, C.M., y De Carvalho, M. (2016). La educación a distancia virtual: desarrollo y características en cursos de matemáticas. Revista Virtual Universidad Católica del Norte, (48), 33-55.

http://www.redalyc.org/articulo.oa?id=194245902004

https://www.redalyc.org/pdf/1942/194245902004.pdf

Tobón, B., Tobón, S., Veytia, M.G., y Escudero, A. (2018). Hacia un nuevo concepto: Plataformas Virtuales Socioformativas ( PVS ). Revista ESPACIOS. ISSN 07981015 Vol. 39 (53).p.27

http://www.revistaespacios.com/cited2017/cited2017-27.pdf

Varela, S., (2020). Uso de las tecnologías de la información y la comunicación como competencia transversal en la formación inicial de docentes. Revista Electrónica Educare (Educare Electronic Journal). EISSN: 1409-4258 Vol. 24(1) Enero-Abril, 2020: 1-20.

https://orcid.org/0000-0001-9994-3209

https://www.scielo.sa.cr/pdf/ree/v24n1/1409-4258-ree-24-01-172.pdf

Vásquez, H. G. (2017). Estudio del Impacto de las TIC en el desarrollo por competencias de los estudiantes de secundaria, en el área de matemáticas de la Institución Educativa Rural El Rosal - Colombia [Tesis de maestría, Universidad Norbert Weiner]. Repositorio Institucional. http://repositorio.uwiener.edu.pe/handle/123456789/1349 
Zempoalteca, B., Gonzáles, J., Barragán, J., y Guzmán, T. (2018). Factors influencing incorporation of Information and Communication Technologies in public universities: approach based on teacher self-perception. Revista de la Educación Superior 47 (186).p.51-74

http://www.scielo.org.mx/pdf/resu/v47n186/0185-2760-resu-47-186-51.pdf 